\title{
Giant Brillouin amplification in gas using hollow-core waveguides
}

\author{
Fan Yang, Flavien Gyger, and Luc Thévenaz \\ Swiss Federal Institute of Technology Lausanne (EPFL), Group for Fibre Optics, \\ SCI-STI-LT Station 11, 1015 Lausanne, Switzerland \\ fan.yang@epfl.ch
}

\begin{abstract}
We report a strong Brillouin amplification in g as u sing hollow-core fibers, exceeding by 6 times the gain in solid silica fibers. A novel fiber laser using Brillouin lasing in gas has been demonstrated. (C) 2020 The Author(s)
\end{abstract}

Introduction - Stimulated Brillouin scattering (SBS) is the strongest nonlinear effect in amorphous materials [1] and has led to many important applications, such as highly coherent laser sources, gyroscopes, slow and fast light, microwave photonic filters, isolators and sensors. SBS has been observed in various platforms, including optical fibers, integrated waveguides, liquids and gases.

Gases offer many attractive features [2] for nonlinear optics because, unlike condensed matter, they are not subject to optical damage at high intensities. In addition, they show pressure-dependent nonlinearity and dispersion, as well as a wider transparency window from vacuum ultraviolet to the mid-infrared region. Various nonlinear effects have been demonstrated in gases.

So far, backward SBS in gases has been exclusively observed using free-space interactions [3, 4]. The weak light confinement over a sizeable interaction length results in limited scattering efficiency, so that high power pump lasers (megawatt peak power) are required to eventually observe a moderate SBS signal [3, 4]. Globally, no strong light-sound interaction in gaseous media has been reported so far.

Here, we report a considerable optical amplification by using backward SBS in a gas-filled hollow-core fiber (HCF), thanks to the quadratic dependence on the gas pressure and the tight confinement of light and gas over long distance. $0.32 \mathrm{~dB}$ of signal amplification per $\mathrm{mW}$ of pump power inside a $50 \mathrm{~m}$ long HCF filled with 41 bar $\mathrm{CO}_{2}$ has been achieved. Using this platform, we demonstrate a low-threshold $(33 \mathrm{~mW})$ continuous-wave single-frequency gas Brillouin laser that can in principle work at any wavelength.

Results - Figure 1(a) shows the measured backward SBS gain spectra of the HCF filled with $\mathrm{CO}_{2}$ at different pressures and, for comparison purpose, of a standard single-mode fiber (SMF) having a solid silica core of similar diameter. The HCF (cross section shown in the inset of Fig. 1(a)) used in this work is a bandgap fiber with a loss of $16 \mathrm{~dB} / \mathrm{km}$ at $1.55 \mu \mathrm{m}$ and with a length of $50 \mathrm{~m}$. The Brillouin gain exceeds that of the SMF for pressures above $\sim 20$ bar. The peak Brillouin gain is proportional to the square of the gas pressure while the Brillouin linewidth (FWHM) is inversely proportional to the pressure. When the pressure reaches 41 bar, the measured Brillouin gain coefficient is $1.68 \mathrm{~m}^{-1} \mathrm{~W}^{-1}$, which turns out to be 6 times higher than in a SMF and 20 times higher than the largest Raman gain achievable in gas-filled HCFs at $1.55 \mu \mathrm{m}$. The Brillouin linewidth for 41 bar $\mathrm{CO}_{2}$ is 3.65 $\mathrm{MHz}$, which is 10 times narrower than in a SMF.

Figure 1(b) plots the measured Brillouin spectra for sulfur hexafluoride $\left(\mathrm{SF}_{6}\right)$, carbon dioxide $\left(\mathrm{CO}_{2}\right)$, nitrogen $\left(\mathrm{N}_{2}\right)$ and methane $\left(\mathrm{CH}_{4}\right)$ under specific pressures. The acoustic velocity, and hence the Brillouin frequency shift, scales inversely to the square root of the gas molar mass, as demonstrated in Fig. 1(b). Mixing gas can be implemented to engineer the Brillouin spectrum.

By scanning the pump-signal detuning frequency, the Brillouin amplification spectra are measured at different pump power. The peak amplification in $\mathrm{dB}$ as a function of pump power inside the HCF is plotted in Fig. 1(c). A slope of $0.32 \mathrm{~dB} / \mathrm{mW}$ has been observed with only a $50 \mathrm{~m}$ long $\mathrm{HCF}$ filled with 41 bar $\mathrm{CO}_{2}$. A record $53 \mathrm{~dB}$ amplification has been observed for a signal input power below $-49 \mathrm{dBm}$ and a pump power of $200 \mathrm{~mW}$ inside the HCF. A straightforward estimation shows that the amplification coefficient could be enhanced to $1.2 \mathrm{~dB} / \mathrm{mW}$ by extending the effective length to $160 \mathrm{~m}$ using the same 41 bar $\mathrm{CO}_{2}$ filled $\mathrm{HCF}$.

This platform can be easily implemented into a gas Brillouin laser by looping the $50 \mathrm{~m}$ long, 41 bar $\mathrm{CO}_{2}$-filled HCF into a fiber ring cavity. Figure 1(d) shows the Stokes power as a function of the pump power evaluated inside the HCF. Brillouin lasing is turned on when the net Brillouin gain exceeds the round-trip loss of the fiber cavity. The measured threshold is $33.2 \mathrm{~mW}$, in good agreement with the theoretically estimated threshold of $34.9 \mathrm{~mW}$. The beating spectrum linewidth between the Stokes signal and a local oscillator (frequency shifted pump) above threshold is measured to be $66 \mathrm{kHz}$, which is much narrower than the spontaneous spectrum. 


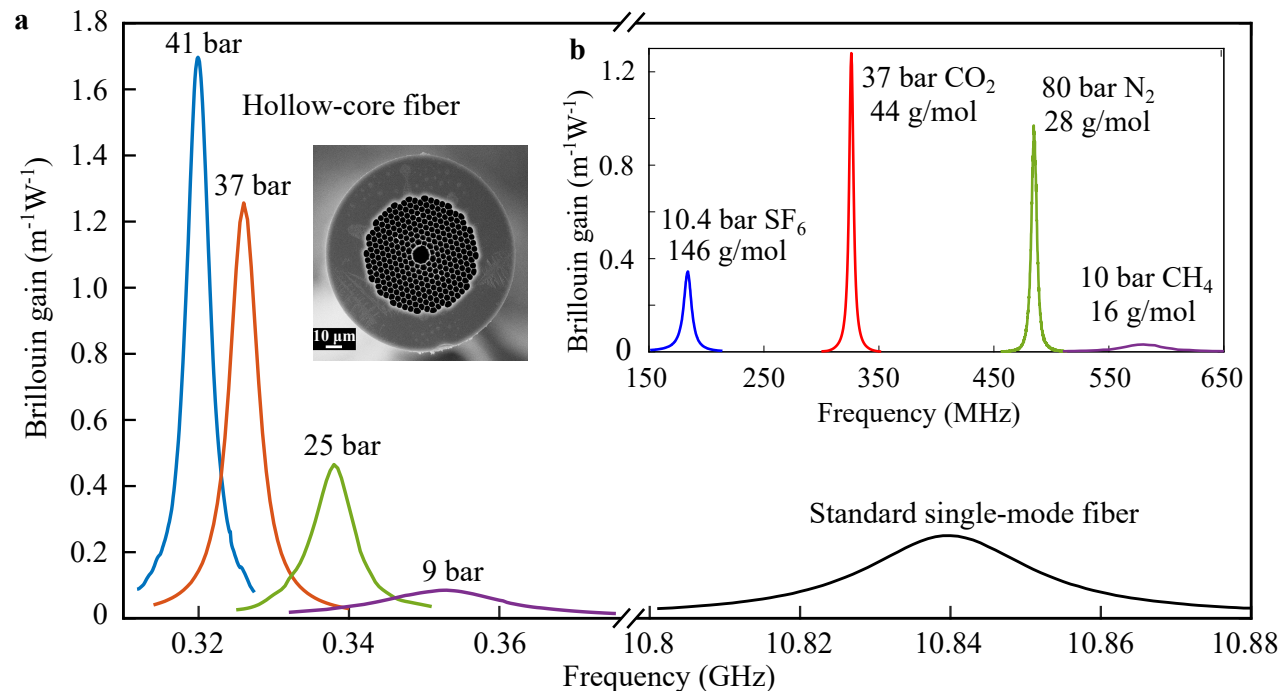

c
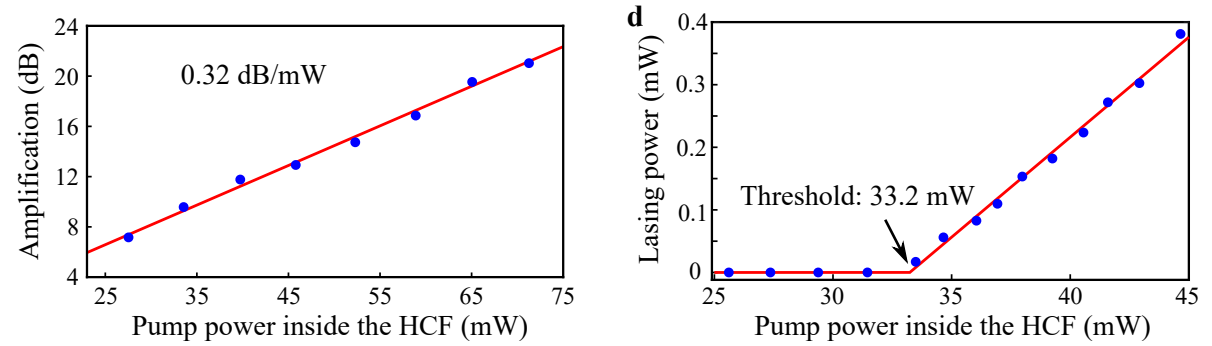

Fig. 1: Stimulated Brillouin scattering in gas-filled hollow-core fibers (HCFs). (a) Measured Brillouin gain spectra in a $\mathrm{HCF}$ filled with $\mathrm{CO}_{2}$ at different pressures. The Brillouin spectrum of a solid silica-core SMF is also shown for comparison. Inset: SEM image of the HCF used in this work. (b) Measured Brillouin gain spectra for 37 bar $\mathrm{CO}_{2}, 80$ bar $\mathrm{N}_{2}, 10.4$ bar $\mathrm{SF}_{6}$ and 10 bar $\mathrm{CH}_{4}$ with the molar mass indicated. (c) Signal amplification experiment: peak probe amplification in logarithmic scale as a function of pump power inside the 41 bar $\mathrm{CO}_{2}$ filled HCF in linear scale. (d) Gas Brillouin lasing experiment: intracavity Stokes power as a function of pump power inside the 41 bar $\mathrm{CO}_{2}$ filled $\mathrm{HCF}$.

Conclusion and discussion - The large light-sound interaction in a gas-filled HCF reported here leads to a measured gain nearly 6 order of magnitude larger than in previous works using free-space optics, 6 times larger than in standard SMF as well as 20 times larger than the highest achievable Raman gain at $1.55 \mu \mathrm{m}$. With this platform, we demonstrate the first low threshold gas Brillouin laser which has not yet been reported due to the extremely low scattering efficiency in free-space implementations.

Our approach offers an efficient way to amplify signals in HCFs, and it can operate at any wavelength from the ultraviolet to the mid-infrared region, limited only by the transmission windows of the HCFs.

This novel gas-based Brillouin platform can be the foundation of many potential applications, some being partially illustrated here: amplifiers, highly coherent Brillouin gas lasers, slow and fast light, microwave filters, tunable delay lines, light storage and sensing. The reduced acoustic loss with respect to silica results in a narrower gain resonance. This turns out to be a clear asset for optical storage, optical signal processing, precisely selective spectral filtering, sensing, etc...

On a broader perspective, the concept introduced in this paper can also be applied to other waveguides. For instance, a small dimension slot waveguide [5], inducing an intense evanescent field can be designed to offer a large light-sound interaction in gas. Hence, if the immediate and massive benefit of Brillouin amplification in gas is undoubtedly for hollow-core waveguides, its potential can certainly extend to other configurations.

Acknowledgement — This work was supported by SNSF under grant agreements No. 178895 and 159897

\section{References}

1. B. J. Eggleton, et al, Nature Photonics 13, 664-677 (2019).

2. P. S. J. Russell, et al, Nature Photonics 8, 278-286 (2014).

3. E. E. Hagenlocker, et al, Applied Physics Letters 7, 236-238 (1965).

4. A. Manteghi, et al, Physical Review Letters 107, 173903 (2011).

5. V. R. Almeida, et al, Optics Letters 29, 1209-1211 (2004). 